\title{
Thermocouple errors in temperature measurements and validation of CFX and FLUENT models of natural gas combustion
}

\section{Pavlo Zasiadko, Mykola Priadko}

National University of food Technologies, Kyiv, Ukraine

\section{Keywords:}

Combustion

Thermocouple

Bead

Heat flux

Simulation

\section{Article history:}

Received 06.09.2016

Received in revised form 16.10.2016

Accepted 27.12.2016

Corresponding author:

Pavlo Zasiadko

E-mail:

iaroslav@nuft.edu.ua

DOI: $10.24263 / 2304-$

974X-2016-5-4-15

\section{Abstract}

Introduction. Technological processes associated with combustion pose serious environmental problems due to the emission of harmful substances predominantly in gaseous state. According to the generally accepted view, the nitrogen oxides mostly originate in combustion plants in the regions of high temperatures.

Materials and methods. Therefore the problems associated with the thermocouple errors at temperature measurements in the flames carried out during experimental combustion of natural gas in the experimental unit VGP-100B are being dealt with.

Results and discussion. A model has been derived aimed at the evaluation of correction factors that are to be applied, when processing data from the direct thermocouples' readings. The model takes into account the convective heat transfer, irradiation on a part of the thermocouple bead, which falls from the combustion products. It also takes into consideration that a part of the thermocouple bead irradiates in the direction of the cooled surrounding. As a result, a transcendent fourth order equation has been obtained and solved numerically by MathCad. A set of correction coefficient was obtained which, when added to the direct readings of thermocouples, have shown a close conformity to the results obtained from the $3-\mathrm{d}$ modeling of the natural gas combustion in the cylindrical down flow reactor.

Conclusion. The developed correction methodology may be recommended to be used in practice, when experimental temperature measurements may be performed by means of bare thermocouples, when sheathed thermocouple or suction pyrometer cannot be used. 


\section{— Processes and Equipment of Food Productions -}

\section{Introduction}

Literature sources $[1-8,10,11]$ deal with the flame temperature measurements with bare thermocouples. They mainly discuss the measurement of temperature in combustion processes within the flame or near in the vicinity of cooled walls or with temperature measurements of relatively cold gas flows in the presence of high-temperature enclosures that are in direct exposure to the thermocouple.

If a thermocouple measures the temperature of gas flow outside of the radiating flame, it most likely gives too high temperature readings, since the thermocouple bead receives not only the convective heat from the flow itself, but radiative flux from the flame as well. Because the radiant flux is proportional to the 4th temperature degree, it is clear that thermocouple measurements in high temperature processes, particularly combustion the input of radiant flux will be much more significant than that from convection.

On the contrary, when measurements take place in a close vicinity of flame with the enclosure kept at a lower temperature and with optically semitransparent medium, the thermocouple readings will be lower than the actual flame temperature. This is due to the effect that the thermocouple bead, being a participant of complex heat transfer, will receive heat as a result of convection from the gas flow and radiation from the flame, will also irradiate towards the cooled enclosure. Shannon and Butler [1] suggested a mathematical model that takes into account the heat balance of the thermocouple bead:

$\left[\varepsilon_{f l} \sigma T_{f l}^{4}-\varepsilon_{\text {bead }} \sigma T_{\text {bead }}^{4}\right] F_{\text {form-bead }}-\left[\varepsilon_{\text {bead }} \sigma T_{\text {bead }}^{4}-\varepsilon_{\infty} \sigma T_{\infty}^{4}\right]\left(1-F_{\text {form bead }-\infty}\right)=h A_{\text {bead }}\left(T_{f l}-T_{\text {bead }}\right)$

where $\mathrm{F}$ - a function that takes into account the relative exposure, flame-bead or bead surrounding, respectively.

In [2] an extremely simplified model was accepted, according to which the thermocouple radiates all the heat, obtained by the convection from the flow:

$$
\left(T_{f l}-T_{\text {bead }}\right)=\frac{\sigma \varepsilon}{h}\left(T_{\text {bead }}^{4}-T_{\infty}^{4}\right)
$$

where $\mathrm{h}$ - heat transfer coefficient from the gas flow calculated by:

$$
N u\left[\frac{\frac{1}{2}\left(T_{f l}-T_{\text {bead }}\right)}{T_{\text {bead }}}\right]^{-0.17}=0.24+0.56\left(\frac{w d}{v}\right)^{0.45},
$$

Combining (2) and (3) one can approximately determine the thermocouple error from the re-radiation:

$$
\left(T_{f l}-T_{\text {bead }}\right) \approx \frac{d^{0.55}}{w^{0.45}}\left(T_{\text {bead }}^{4}-T_{\infty}^{4}\right) .
$$

Where- $w$-gas flow speed, may be termed as a portion of heat delivered by convection. Some conclusions are readily available from this correlation: the reduction of the error can be achieved by reducing the diameter of the thermocouple bead or by decreasing the proportion of radiant heat transfer between the gas flow and the bead. 


\section{— Processes and Equipment of Food Productions -}

Walker-Stokes [3] proposed the method of measuring the temperature with thermocouple set, whose diameter is decreased progressively. This method has allowed to reduce inertia of thermocouple readings and to determine the effect of radiation basing upon the expression (4). This method allows also to increase the accuracy of measurements, but its implementation is too complex, because it requires simultaneous temperature measurement with the range of thermocouples of different sizes. The method becomes absolutely unrealizable due the temperature instability of flows and necessity to install the thermocouple in the same place of a flow.

Most often, the protective screens are used to shield thermocouple beads [1-4]. Since the protective screen has an intermediate temperature between the actual temperature of bead and the ambient temperature, it significantly reduces the re-radiation flux. Protective screens can be accompanied with the suction of flow through a cylindrical screen, which leads to an increase of flow speed around the thermocouple bead and, as a result, increasing convective heat transfer coefficient thus bringing closer bead actual temperature to that of flow. In practice, the speed of sucked flow is limited by the experimental considerations (prevention of significant disturbance in the flow). Comparative results of the temperature measurement in industrial furnaces by means of unprotected thermocouples of different diameters, two types of shielded thermocouples, that differed by the place of suction, were given in $[1,2,8]$. The authors have shown that the greatest error occurs at measuring the temperature of medium with the thermocouple irradiation.

From the data given in [1-8] follows that the error of "cold" flow measurement by bare thermocouple within the radiating environment reaches $250 \%$. Errors of temperature measurements by bare thermocouples in "hot" flows surrounded by cooled walls reach $25 \%$. Installing of a dual screen can reduce the error to $25 \%$ and $7 \%$.

\section{Materials and methods}

Experiments have been conducted in the experimental rig designed and erected at the Coal Energy Technology Institute of the National Academy of Science of Ukraine. The scope of experimentation covered the following:

- Obtaining experimental data of the direct temperature and components" concentration distribution within the reactor when burning natural gas, which then will be used for the 3-D CFX and FLUENT models validation by the comparison of the measured and modeled data;

- Co-combustion of coal and solid biomass aimed at the determination of the optimal process conditions;

- Combustion of solid biomass at different regime parameters aimed at the determination of the effect of the various types of biomass burning kinetics, its conditions and properties aimed at the determination of the optimal process conditions.

Since the experimental rig closely models the conditions which exist in the lower radiation section of industrial boiler furnaces in the vicinity of burners, the experimental results obtained thus may be applicable for the designing optimal combustors.

The schematic (layout) of the stand is shown in Figure 1.

Thermal capacity of the unit when burning coal with air as an oxidant reaches 100 KW. 

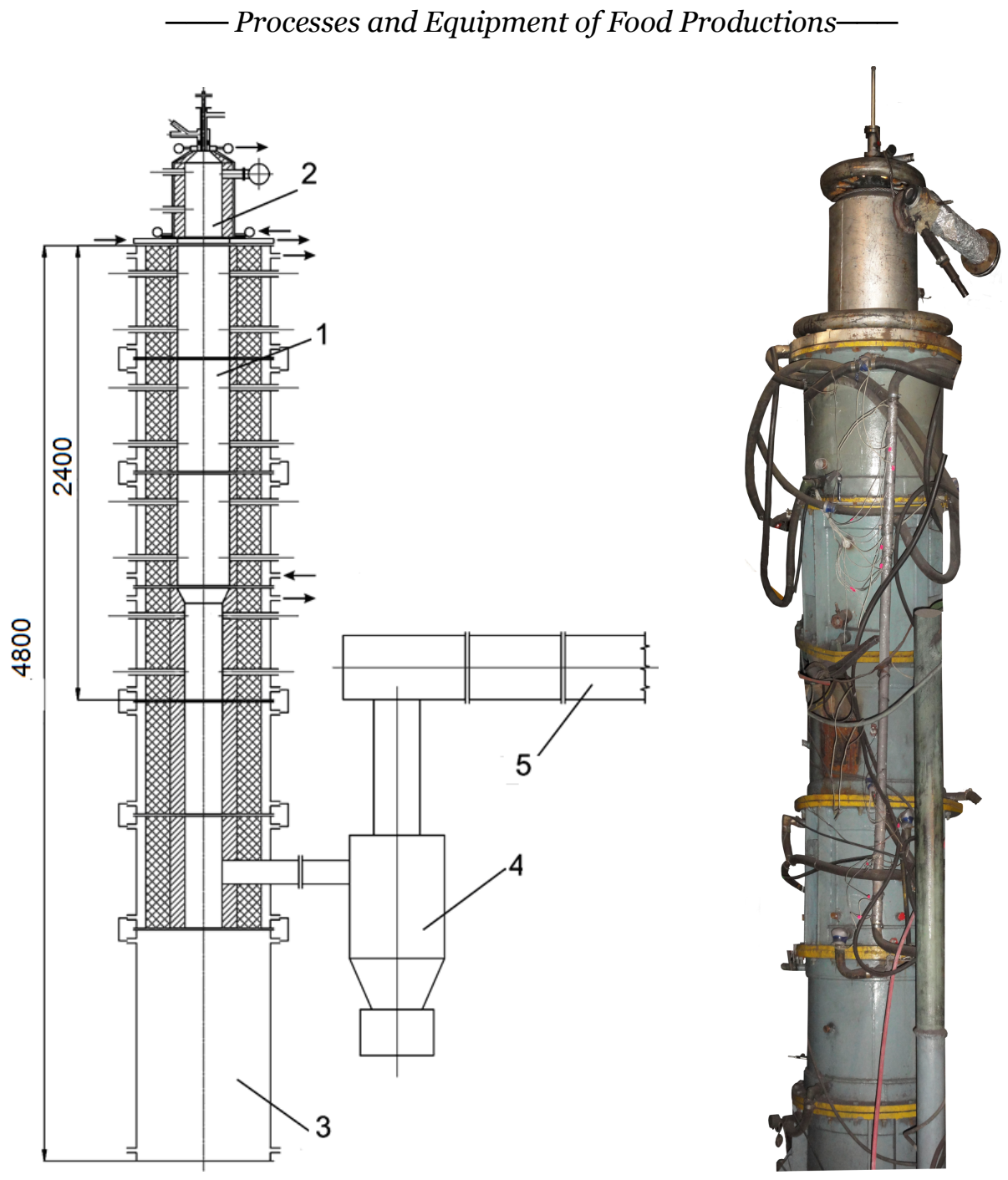

Figure 1. Experimental down flow reactor VGP-100 B:

1 - experimental sections, water cooled jackets for calorimetry;

2-Burner head with multiple air inlets;

3-ash collector;

4-cyclon for fly ash removal;

5-flue gases duct.

The main part of the unit is a down flow test channel with four diagnostic sections in which combustion takes place. Each of three initial sections is $0.6 \mathrm{~m}$ long with the internal diameter $0.28 \mathrm{~m}$. The last one has the same length and $0.2 \mathrm{~m}$ internal diameter. The internal walls of all test sections have a tree layer protective covering consisting of consecutive 


\section{— Processes and Equipment of Food Productions -}

layers of zircon dioxide, fire clay and asbestos. Each section has a water cooled jacket with the individual supply and water flow measurement, which in turn allows the calorimetry of each section. The burner head is equipped with two natural gas burners. The main burner has an additional channel for the injection of pulverized (ground) solid biomass or coal together with NG and initial air. The secondary burner is designed to inject natural gas and air only. There are also additional inlets allowing to inject any of the combustion components with the respective measurements of the component's flow rate. Each section also equipped with the inlets allowing inserting probes and thermocouples allowing direct measurements of local temperature and flue gases composition.

The experiments of natural gas combustion were carried out in the down flow cylindrical reactor VGP-100, Figure 1. The temperature of test sections' walls was measured by chromel-alumel (type K) thermocouples that were built in the fire proof lining $10 \mathrm{~mm}$ deep from the fire layer. Periodical measurements of fire layer of lining were performed with the pyrometer "DPR-1" through the diagnostic openings. The temperature of the gas flow on the axis of the reactor was measured by Platinum-Platinum-Rhodium thermocouples (Type R) with $0,2 \mathrm{~mm}$ diameter wires in corundum protective cover with $0,5 \mathrm{~mm}$ diameter channels positioned in probes at a midsection of each test section. Hot thermocouple junctions were covered with a layer of corundum making the bead diametr-5 $\mathrm{mm}$.

Thus obtained data related to the combustion of natural gas of a known composition were used for the validation of the developed 3-D CFX model of the process along with the direct temperature measurements which were used as benchmark data for developed model of measured temperatures correction.

In order to carry out the validation, a complex 3-d meshes of the reactor has been developed. The mesh represents the experimental stand VGP in general and in minor details. The main problem of the mesh development consisted in finding an optimum compromise between the number of mesh cells (which eventually determines accuracy and calculations convergence time and, thus, the required computer capacity) on the one hand, and the necessity to represent minor stand details which affect the actual process and are to be represented in the mesh - on the other.

Combustion of methane in down flow cylindrical reactor VGP-100B of the Coal Energy Institute of the National Academy of Sciences of Ukraine (ICE NASU) has been studied. The temperature inside the reactor was measured as follows -temperature of walls was measured by chromel-alumel (type K) thermocouples that were built in the fire proof lining $5-10 \mathrm{~mm}$ deep from the fire layer with continuous registration of temperature. Periodical measurements of fire layer of lining were performed with the pyrometer "DPR$1 "$ through the diagnostic openings. The temperature of the gas flow on the axis of the reactor was measured by thermocouples, Platinum-Platinum-Rhodium (Type R) with 0,2 $\mathrm{mm}$ diameter wires embedded in corundum protective cover with $0,5 \mathrm{~mm}$ diameter channels and put through the diagnostic openings. Hot thermocouple junctions were covered with a layer of corundum making the bead diametr-5 mm. Lack of thermal stabilization of cold junctions was accounted for by correction factor for a local air temperature. The process has been modeled in a 3-d arrangement with the CFX-15 and FLUENT (Lic.No1023420 ) commercial codes. 


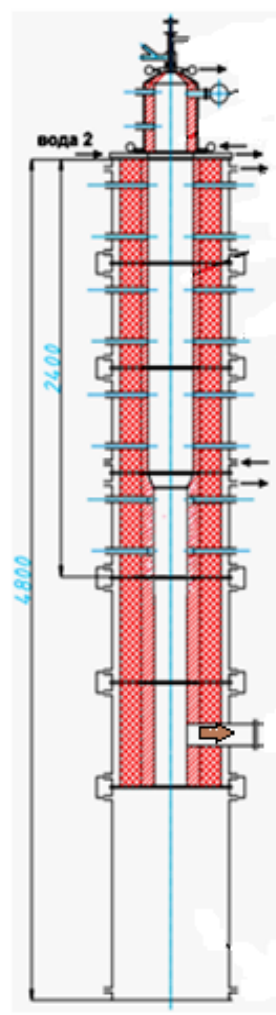

a

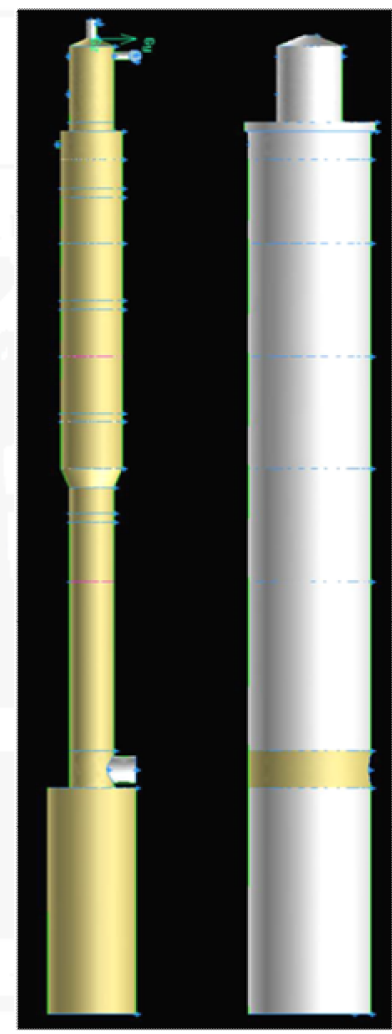

b
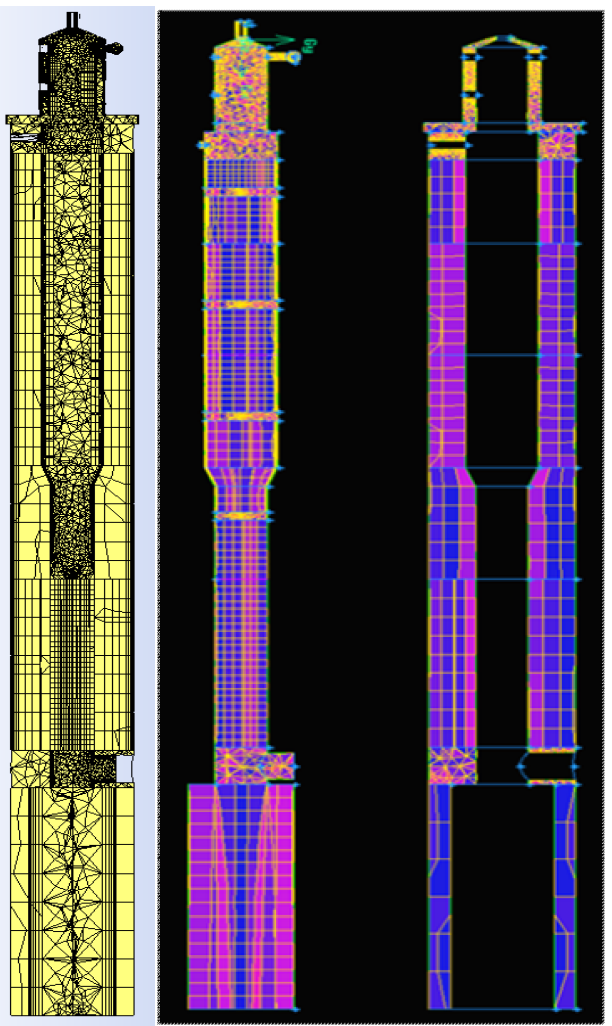

$\mathrm{c}$

Figure 2. Reactor VGP:

a - VGP Reactor, $b$ - geometry model with and without insulation, c-3-D mesh of the reactor core and insulation

\section{Results and discussion}

The results of simulation with CFX and FLUENT software, particularly the temperature distribution along the reactor axis, heat fluxes from the diagnostic sections were compared to those observed experimentally.

It was found that the gas temperature measured in 4 points in the middle of each diagnostic section on the axis were $120-150{ }^{\circ} \mathrm{C}$ lower than respective CFX-FLUENT simulated values. The difference in the obtained data can be explained by re-radiation of thermocouple beads towards the cooled walls. Thermal balance of thermocouple bead, that considers both convective and radiant flows, can determine the value of error of flow temperature measurement on the axis of the reactor.

It should be noted that the model under consideration becomes significantly complex as a result of taking into account radiant heat transfer between the thermocouple and gas flow, since gases are characterized by selective spectral energy absorption and emission. The absorption and emission of energy by monatomic and diatomic gases, including nitrogen, 
oxygen, and hydrogen are insignificant and may be neglected. Polyatomic gases, including carbon dioxide $\mathrm{CO}_{2}$, water vapour $\mathrm{H}_{2} \mathrm{O}$, sulphur dioxide $\mathrm{SO}_{2}$, ammonia $\mathrm{NH}_{3}$, and others, have a large capacity for absorption and emission of radiant energy. Carbon monoxide $\mathrm{CO}$ also has a significant level of emission and absorption, but this gas occurs only as traces in the products of methane combustion at a proper air-fuel ratio.

The model of radiant heat exchange of bead with the environment has been derived under the assumption that the gas has a constant temperature $T_{f l}$, and the wall has a constant temperature $T_{\text {wall }}$.

Flue gas and the walls are considered gray bodies. Radiation of the wall is characterized by a continuous spectrum and the medium (combustion gases) has selectively radiation in a form of separate bands $\mathrm{e}_{1}, \mathrm{~g}_{1} ; \mathrm{e}_{2}, \mathrm{~g}_{2}$. In the general case the number of such bands varies.

Radiation heat transfer from the gas to the wall can be expressed as:

$$
Q_{f l-\text { wall }}=\left(E_{e f . f l-\text { wall }}-E_{e f . \text { wall }-f l}\right) A_{\text {wall }},
$$

where $A_{\text {wall }}-$ surface area of the wall;

$E_{\text {ef.fl-wall }}, F_{\text {ef.wall-fl }}$ - effective radiation heat flux from gas medium and the effective radiation of the wall, respectively.

The effective radiation heat flux of the gas medium and the walls was calculated based upon the balance method. The values can be represented by the expressions that are valid within certain radiation spectral bands $[9,13]$ :

$$
\begin{gathered}
E_{e f . f l}=\left(E_{0 f l}\right)_{\Delta \lambda}+q_{f l-\text { wall }}\left(1-\frac{1}{\varepsilon_{f l, \Delta \lambda}}\right), \\
E_{e f \text { wall }}=\left(E_{0 \text { wall }}\right)_{\Delta \lambda}+q_{\text {wall }-f l}\left(1-\frac{1}{\varepsilon_{\text {wall }, \Delta \lambda}}\right) .
\end{gathered}
$$

The fractions of black body emission of the gas and wall at the limiting values of their emissivity factors can be expressed by the following expressions:

$$
\begin{gathered}
\left(E_{0, f l}\right)_{\Delta \lambda}=C_{0}\left(\frac{T_{f l}}{100}\right)^{4} \cdot \varepsilon_{f l}^{\infty}, \\
\left(E_{0, \text { wall }}\right)_{\Delta \lambda}=C_{0}\left(\frac{T_{\text {wall }}}{100}\right)^{4} \cdot \varepsilon_{\text {wall }}^{\infty},
\end{gathered}
$$

where $\varepsilon_{f l}^{\infty}$ and $\varepsilon_{\text {wall }}^{\infty}$ - are limit values of emissivity factors for gas and walls, respectively, which in case of the wall radiation assumes the black body radiation at the wall temperature, whereas in case of gas radiation it is assumed that the optical length of the gas layer approaches infinity, i.e. gas radiates at its spectral bands as a black body. 


\section{- Processes and Equipment of Food Productions-}

The values are determine from the charts [12] at temperatures of gas and wall, respectively. Gas emissivity $\varepsilon_{f l, \Delta \lambda}$ is determined by the following:

$$
\varepsilon_{f l, \Delta \lambda}=\frac{k_{1} l_{1} e_{1} g_{1}+k_{2} l_{2} e_{2} g_{2}}{a_{1} b_{1} e_{1} g_{1}+a_{2} b_{2} e_{2} g_{2}}=\frac{E_{f l, \Delta \lambda}}{\left(E_{0, f l}\right)_{\Delta \lambda}}=\frac{\varepsilon_{f l} E_{0, f l}}{\varepsilon_{f l}^{\infty} E_{0, f l}}=\frac{\varepsilon_{f l}}{\varepsilon_{f l}^{\infty}}
$$

Equation (8) can be interpreted by means of Figure 3. As it can be seen, $J_{b b}, J_{g r}$ are lines that depict spectral radiation intensity of black and "grey" body, respectively at a certain temperature.

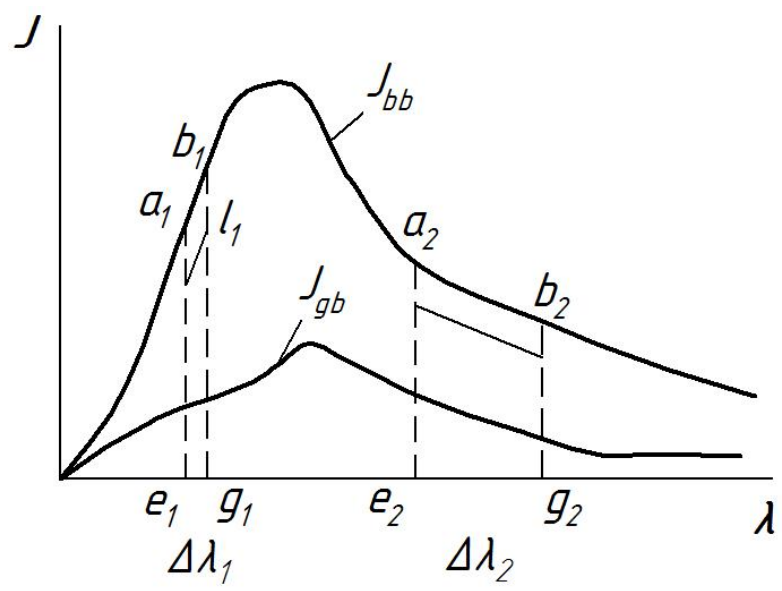

Figure 3. Spectral radiation intensity of black, grey bodies and selective radiation of gas

If one suggests that a certain gas radiates within two bands $\Delta \lambda_{1}, \Delta \lambda_{2}$ and energy emitted within these bands somewhat lower than that of the black body, namely those equal graphically to the area within the bands $e_{1} k_{1} l_{1} g_{1}$ and $e_{2} k_{2} l_{2} g_{2}$.

At the same time, within the same bands the black body radiates energy that can be depicted by area $e_{1} a_{1} b_{1} g_{1}$ and $e_{2} a_{2} b_{2} g_{2}$, see Figure 3 . Therefore, the gas emissivity (absorptivity) within the bands $\Delta \lambda_{1}, \Delta \lambda_{2}$ can be calculated by (8), where $\varepsilon_{f l}^{\infty}$ can be interpreted as the emissivity (absorptivity) of a gas layer with infinite thickness within a whole range of wave length $\lambda$. When taken within all spectral bands the gas radiates, this value will be equal to the fraction of black body radiation.

The degree of wall emissivity within certain bands $\Delta \lambda$ can be considered as equal to integral emissivity factor $\varepsilon_{\text {wall, } \Delta \lambda}=\varepsilon_{\text {wall }}$. For the stationary conditions regime holds the following $q_{f l-w a l l=-} q_{\text {wall-fl } l}$.

Substituting $(7,8)$ into $(6)$, we obtain the following expression for the radiant flux $Q_{2, c}$, that is transmitting from the gas medium to the thermocouple bead: 


$$
Q_{f l \text {-wall }}=\frac{C_{0} A_{b}\left[\varepsilon_{f l}^{\infty}\left(\frac{T_{f l}}{100}\right)^{4}-\varepsilon_{f l, \text { wall }}^{\infty}\left(\frac{T_{\text {wall }}}{100}\right)^{4}\right]}{\frac{\varepsilon_{f l}^{\infty}}{\varepsilon_{f l}}+\frac{1}{\varepsilon_{\text {wall }}}-1} .
$$

Integral values of emissivity factor (absorption coefficient) for a gas mixture, as it was shown above, in general case is not equal to the sum of the values of the individual components of the mixture. Thus, for a mixture of $\mathrm{CO}_{2}$ and $\mathrm{H}_{2} \mathrm{O}$ the emissivity factor and the absorption coefficient are less than the sum of their values for $\mathrm{CO}_{2}$ and $\mathrm{H}_{2} \mathrm{O}$ separately, due to its partial overlapping of emission spectral bands:

$$
\varepsilon_{f l}=\varepsilon_{H 2 O}+\varepsilon_{C O 2}-\Delta \varepsilon_{f l},
$$

The individual values of steam and carbon dioxide emissivity were taken from $[12,13]$ at gas temperature and corresponding component's partial pressure in the gas mixture, and beam path length, $p l$. Average path length of the beam was determined from the following expression:

$$
l=m \frac{4 V}{A}
$$

where $V$ is a volume of a gas body;

$\mathrm{m}=0,9$ - correction factor.

The results of direct temperature measurements with bare thermocouples during the experimental combustion of natural gas in a vertical reactor VGP-100B (100 KW th) of the Coal Energy Technology Institute were taken as benchmark values for the validation of the model. The objective of the proposed model is to derive a comparatively simple equation which will allow to calculate the temperature of a hot bead of a thermocouple ( $\left.T_{t h}\right)$ placed into the flow of hot combustion gases at a given $\left(T_{g}\right)$ and being exposed to the relatively cold walls at given $T_{w}$. Thus, the correction temperature can be determined which is to be used when direct readings of unsheathed thermocouples which are used for temperature measurements in media with convective-radiative heat transfer.

The equation was derived under the condition of equality of heat supplied to a thermocouple bead and the amount of heat, that thermocouple gave off to the walls as a result of re-radiation. Heat input is realized by means of convective heat transfer, radiation of hot flue gas and by radiation of heated head burner lining.

For the approximate estimation of the re-radiation effect it was decided to apply the simplified model of radiant heat transfer between the bead surface and the heated lining surface under the following conditions: a) bead surface and radiating lining surface are plane-parallel; b) the area of bead surface, that receives radiation, is equal to a half of the total area of thermocouple bead; c) absorbing capacity is $\varepsilon_{e f}=0.9$; d) the temperature of the heated lining is taken equal to the temperature of the gas core $T_{f l}$. Then, the components of a heat balance of the bead will be: 
Absorbed heat

$Q_{\text {bead abs }}=h\left(T_{f l}-T_{b}\right) A_{b}+\frac{\varepsilon_{e f} A_{b}\left[\left(\frac{T_{f l}}{100}\right)^{4}-\left(\frac{T_{b}}{100}\right)^{4}\right]}{2}+\frac{C_{0} A_{b}\left[\varepsilon_{f l}^{\infty}\left(\frac{T_{f l}}{100}\right)^{4}-\varepsilon_{f l, t \text { wall }}^{\infty}\left(\frac{T_{b}}{100}\right)^{4}\right]}{\frac{\varepsilon_{f l}^{\infty}}{\varepsilon_{f l}}+\frac{1}{\varepsilon_{\text {wall }}}-1}$

Heat, radiated by the thermocouple bead on the wall

$$
Q_{\text {bead }, \text { rad }}=\varepsilon_{c} A_{b}\left[\left(\frac{T_{b}}{100}\right)^{4}-\left(\frac{T_{\text {wall }}}{100}\right)^{4}\right]
$$

where $T_{b}, T_{\text {wall }}, T_{f l}$ - temperatures of thermocouple, wall, actual temperature of the gas, respectively, $[K]$;

$h$ - heat transfer coefficient from the thermocouple bead surface to gas environment;

$\varepsilon_{f l}$ - emissivity factor of gas;

$\varepsilon_{f l}^{\infty} \varepsilon_{f l, t \text { wall }}^{\infty}$ - limiting emissivity factor of gas at $1=\infty$, gas at the temperature of gas, and gas at temperature of the wall, respectively;

$\varepsilon_{c}-$ emissivity factor of corundum,

$A_{b}$ - surface area of the bead.

The heat transfer coefficient was calculated for the case of an external flow of hot flue gases around a thermocouple. The correlation for determination of $N u$ number was taken from [9] and thermophysical parameters were calculated by the software EnecCalc3, and checked with data base [9]:

$$
\overline{N u}=2+0.03 \operatorname{Re}^{0.54} \operatorname{Pr}^{0.33}+0.35 \operatorname{Re}^{0.58} \operatorname{Pr}^{0.36} .
$$

The speed of the flue gases for determining the Reynolds number was calculated on the basis of process modeling CFX 15 Fluent code (Lic.No 1023420) and conventionally accepted as constant $\mathrm{w}=4.5 \mathrm{~m} / \mathrm{s}$. The diameter of the thermocouple bead accepted at $\mathrm{d}-0.005$ $\mathrm{m}$. The values of heat transfer coefficients were calculated for three different temperatures of incoming flow - flue gases core.

The calculations were performed with variations of temperatures of the gas core within the limits $\boldsymbol{T}_{f l}=2200 \ldots 1600 \mathrm{~K}$ and with the temperature of cooled walls variation so that the temperature difference between the wall-flow changed recursively $200 \ldots 400 \ldots 600 \mathrm{~K}$, which corresponds to the actual conditions of the experiment. Thermophysical parameters of combustion products, optical properties of $\mathrm{CO}_{2}$ and $\mathrm{H}_{2} \mathrm{O}$, both individually and in mixtures were defined as a result of the process modeling). Convective heat transfer coefficients were calculated for each pair of the assumed combustion gas and walls temperatures, accordingly.

Thus derived transcendental equation of 4th degree with nonlinear coefficients was solved in Mathcad software package as the point of intersection of the two functions (11 and 12). The solution was obtained in graphical and numerical forms.

The results of equations solving for different cases of gas stream and wall temperatures are summarized in Table 1 and shown in the graph (Figure 4). 
Table 1

Summary table of thermocouple readings at different temperatures of the gas core and walls

\begin{tabular}{|c|c|c|}
\hline $\boldsymbol{T}_{\boldsymbol{f l}}, \mathbf{K}$ & $\boldsymbol{T}_{\text {wall }}, \mathbf{K}$ & $\boldsymbol{T}_{\text {bead }}, \mathbf{K}$ \\
\hline 2200 & 2000 & 2077 \\
\hline & 1800 & 1986 \\
\hline & 1600 & 1910 \\
\hline & 1400 & 1854 \\
\hline & 1200 & 1814 \\
\hline 2000 & 1800 & 1880 \\
\hline & 1600 & 1792 \\
\hline & 1400 & 1724 \\
\hline & 1200 & 1677 \\
\hline 1800 & 1600 & 1686 \\
\hline & 1400 & 1606 \\
\hline & 1200 & 1548 \\
\hline
\end{tabular}

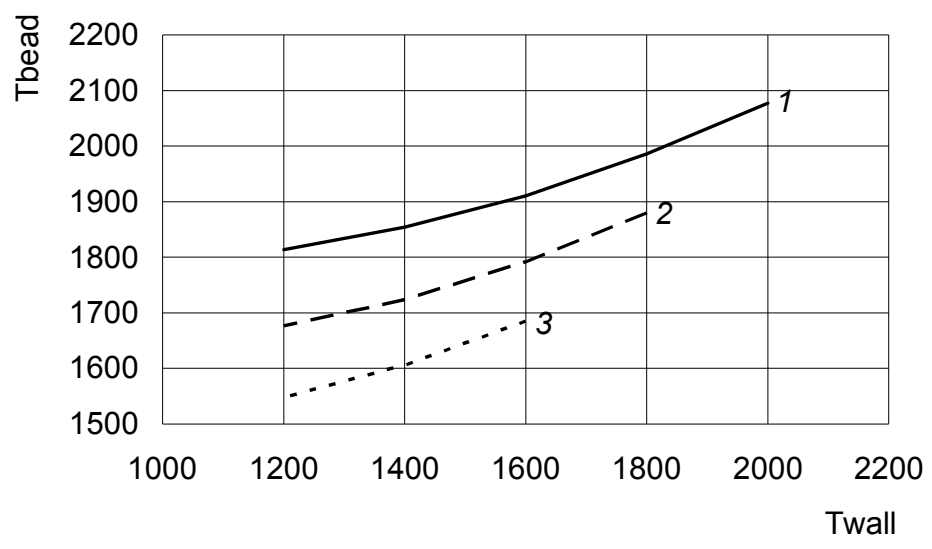

Figure 4. Calculated readings of a thermocouple installed in the flow of flue gases and emitting radiation on the channel wall $\left(T_{\text {wall }}\right)$ at different temperatures of a flow $\left(T_{f}\right)$ : 1 - $T_{f l}=2200 \mathrm{~K}, 2-T_{f l}=2000 \mathrm{~K} ; 3 T_{f l}=1800 \mathrm{~K}$.

According to the obtained data, shown in the Figure 1, at the actual temperature of the flow $2200 \mathrm{~K}$ and $2000 \mathrm{~K}$ temperature of the wall, thermocouples will show the temperature at $123 \mathrm{~K}$ less than the actual gas temperature. At the same gas temperature, the difference between actual temperature and measured thermocouple will increase to $386 \mathrm{~K}$ at $1200 \mathrm{~K}$ temperature of the wall. At $1800 \mathrm{~K}$ of core temperature and $1600 \mathrm{~K}$ of wall temperature specified difference will be $114 \mathrm{~K}$ that is slightly smaller than the measurement of a core temperature.

Analyzing the obtained data, one can conclude that re-radiation plays a significant role in the measurement of temperature at the considered conditions. The graphical interpretation of Mathcad solution of the developed model at a flow temperature of $1400 \mathrm{~K}$ and variations of wall temperature is presented in Figure 5. 


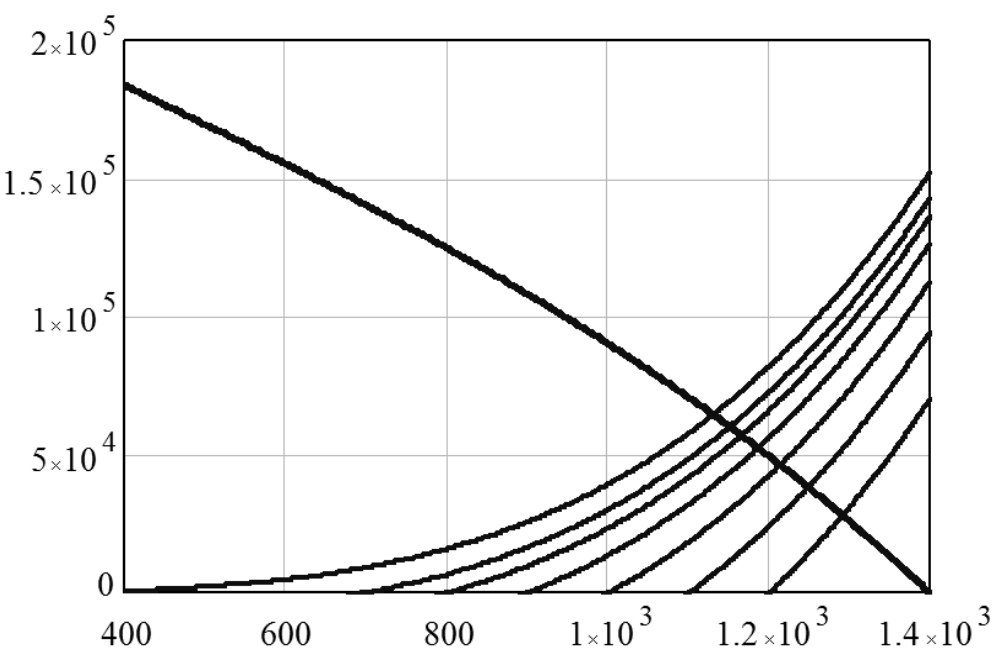

Figure 5. Calculation by the model for conditions $[2,8]$

Each abscissa value of the lines' intersection gives the value of thermocouple bead temperature at the flue gas temperature $1400 \mathrm{~K}$ and respective wall temperature. The bigger the wall temperature, the closer to the $1400 \mathrm{~K}$ value moves the intersection point, and respectively closer to $1400 \mathrm{~K}$ moves the reading of the thermocouple.

To define a degree of reliability of the developed model, the data obtained from the above calculation were displayed in coordinates given in $[2,8]$ namely, $\mathrm{T}_{\text {error }}$ - difference between the gas temperature and thermocouple bead temperature as a function of wall temperature $T_{\text {wall }}$. The result are given in of Figure 6.

Apparently, the data obtained from the proposed model are slightly lower than those given in $[2,8]$ at wall temperatures below $850 \mathrm{C}$. This can be explained by the fact that in our model it is assumed that the bead emits energy by the half of its surface that is directed down towards the ash collector, as the upper part of the bead is directed to head with burners, which emit the energy to the bead. In addition, the proposed model takes into account emission and absorption of triatomic gases in the combustion products, whereas the models threat the gas flow as a transparent medium.

It seems possible to use the obtained data to adjust the results of a direct temperature measurement of the thermocouples located at stand VGP-100D, and to compare them with the calculated data of the CFD modeling process by means of FLUENT and CFX packages. The comparison of direct measurement readings and with those determined by the 3-d model and proposed correction are shown in Figure 7. Data in Figure 7 show that the readings of a direct temperature measurement by thermocouples located along the axis of the channel of the reactor VGP 100B and respective temperatures determined on the basis of simulation are significantly differ. This happen because the measurements by thermocouples are taken under the condition of reradiation from the thermocouples to the cooled walls toward downstream direction. Correction of thermocouple readings was based on the model described above. Thus, the calculation of correction factors was based on the stream temperatures according to the model. 


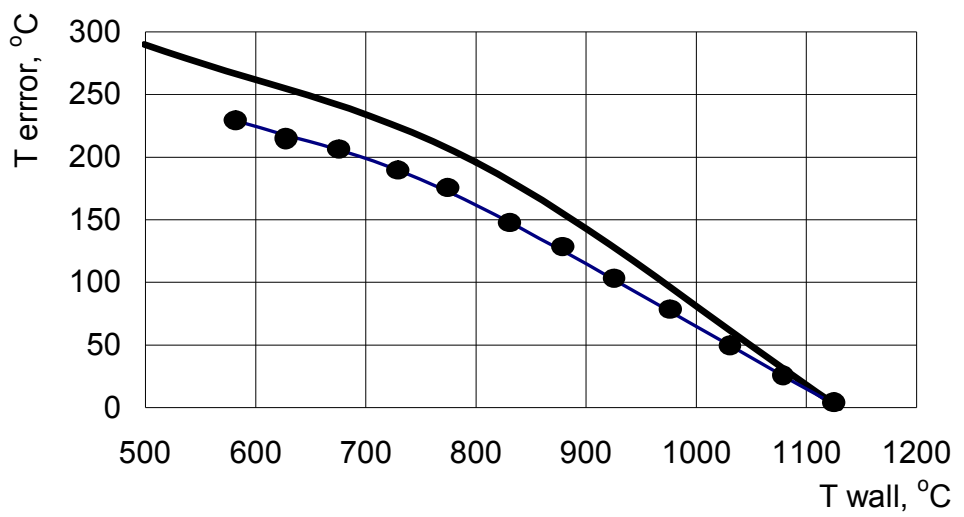

Figure 6. Comparison of the calculations based upon the developed model and data $[2,8]$. The solid line are data $[2,8]$. The points - calculation by the model.

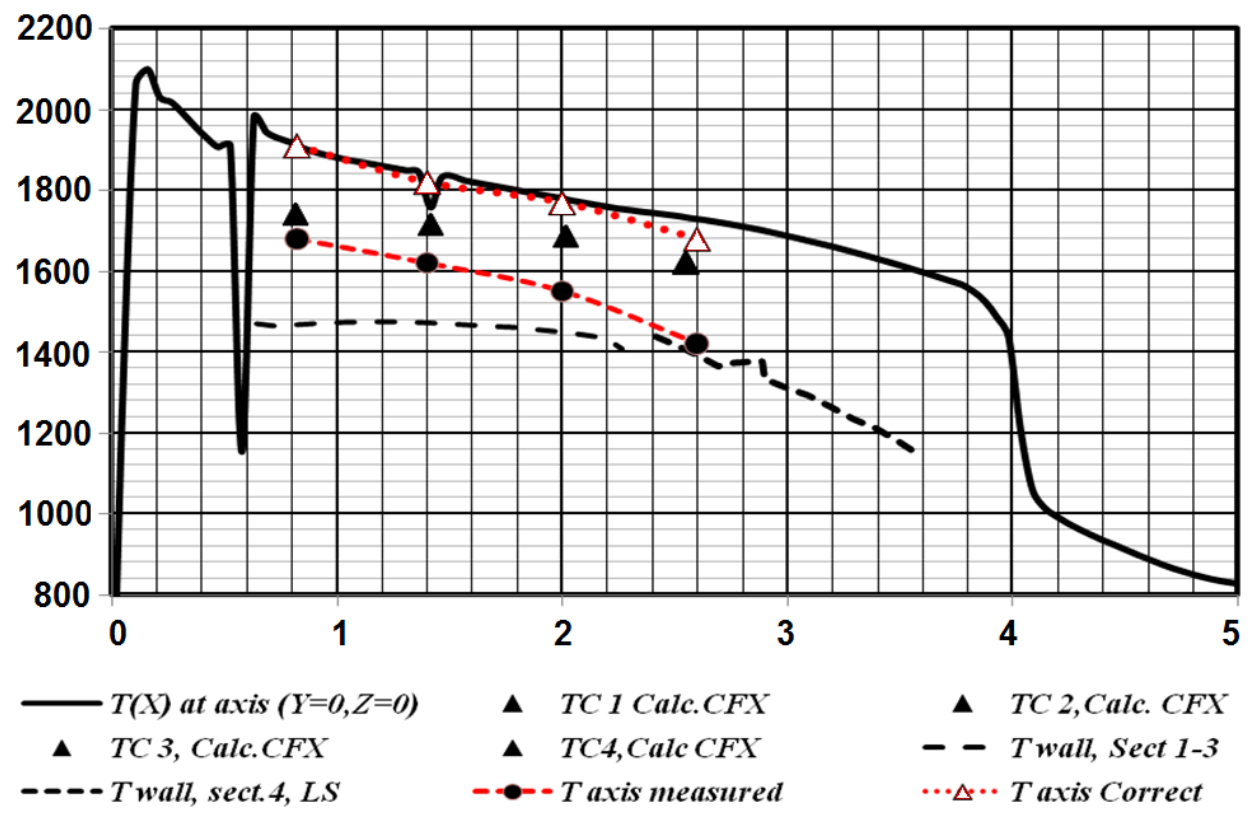

Figure 7. Comparison of the results of calculation by means of CFX package with the results of measurements with a consistent refinement that based on the model of reradiation accounting

The temperature of the wall was taken according to the measurements that were considered reliable because the wall thermocouples, being embedded in the lining, do not participate in radiation heat transfer, and their readings are deemed as reliable. Thus, obtained corrections were added to the thermocouple readings because they reflect the bead 
temperature and not the temperature of the flow of combustion products that flows around the thermocouple bead. Instead, corrections to the thermocouple readings reflect the temperature of stream that flow around beads, see equation (11.12).

As it can be seen from Figure 4, based on the readings of thermocouples and proposed model of measuring the temperature of the flow on VGP channel axis matched with the modeling results close enough (within 5\%), which indicates adequacy of the proposed model and accuracy of data obtained by measurements by the thermocouples. In general, the comparison of the results of CFX simulation of the process of natural gas burning in VGP stand by expendable and operational parameters that correspond to the results of the experiment, indicates their resemblance, which, in turn, is an evidence of a model validation (confirmation of adequacy).

Validation of the models was based on a comparison of heat loss from the parts of VGP stand, that were subjected to calorimetric measurement and losses, that were deducted on the CFX model basis.

Table 2

Comparison of heat losses on the calorimetric stand sections

\begin{tabular}{|c|c|c|c|}
\hline $\begin{array}{c}\text { Section of the } \\
\text { experimental unit }\end{array}$ & $\begin{array}{c}\text { Heat loss measured } \\
\text { on the stand, W }\end{array}$ & $\begin{array}{c}\text { Heat loss of a } \\
\text { model, W }\end{array}$ & $\mathbf{\Delta , ~ \%}$ \\
\hline Burner & 8600 & 9113 & 5.9 \\
\hline Flange of a burner & 2500 & 2320 & 7.2 \\
\hline Diagnostic section & 4300 & 4641 & 7.3 \\
\hline Total & 15400 & 15738 & 2.2 \\
\hline
\end{tabular}

Apparently, there is a close correspondence of the results within $7.5 \%$ on sections and $2.2 \%$ on stand.

Examples of modeling the processes of natural gas combustion using FLUENT package in VGP stand are shown in Figure s 5-7.

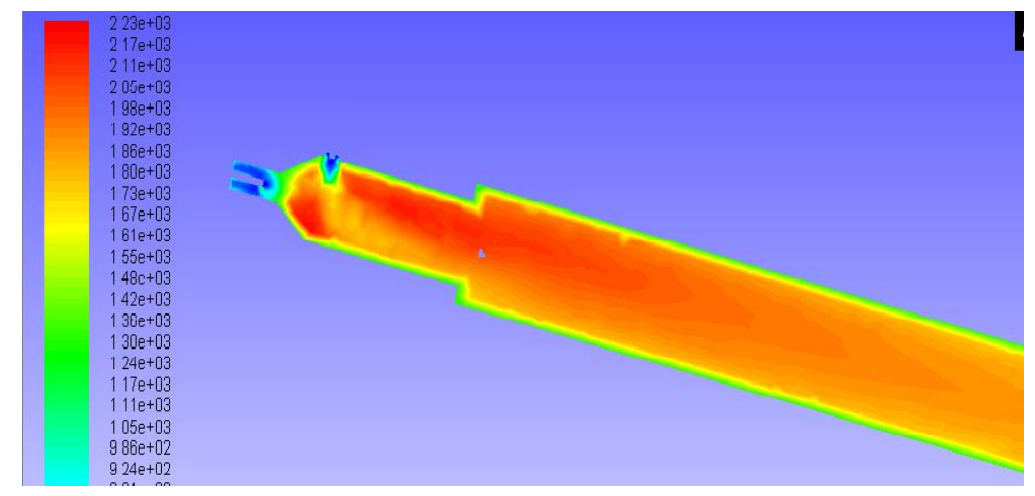

Figure 5. Temperature field in the axial section of VGP with the supply of $125 \mathrm{~m}^{3}$ of air to the burner head 


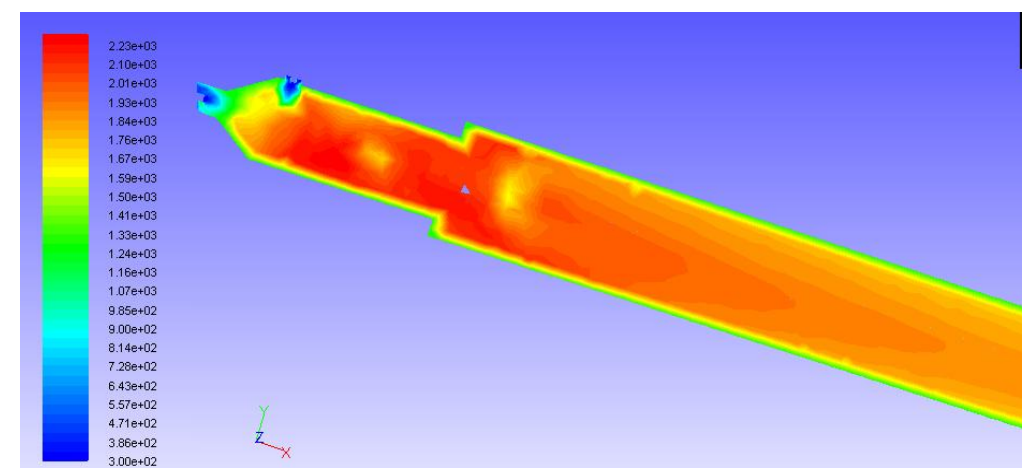

Figure 6. Temperature field in the axial section VGP, mode B 41, supply of $77 \mathrm{~m}^{3}$ of air to the burner head and $48 \mathrm{~m}^{3}$ to the diagnostic section

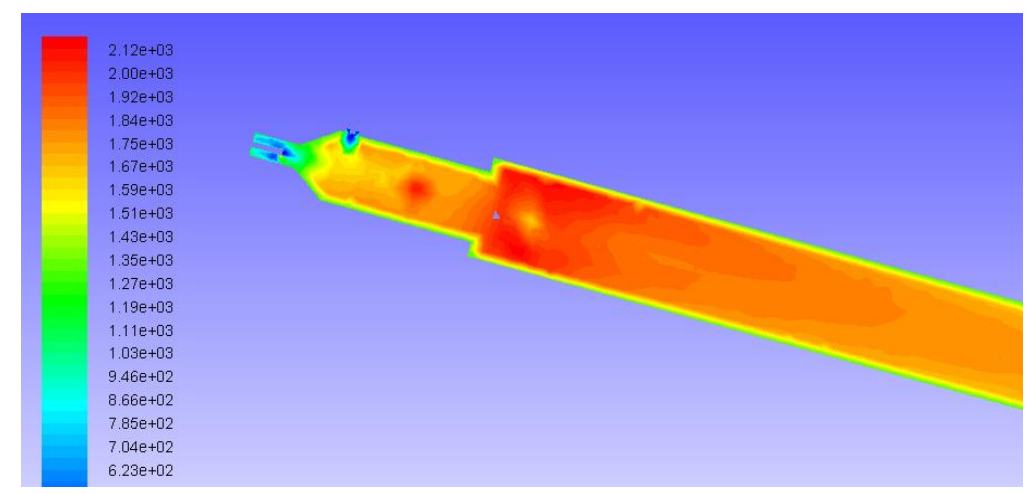

Figure 7. Temperature field in the axial section VGP, supply of $25 \mathrm{~m}^{3}$ of air to the burner head and $100 \mathrm{~m}^{3}$ to the diagnostic section

\section{Conclusions}

1. CFD modeling gives a very powerful tool for the study of combustion processes of different fuels, allowing any variation of operational parameters and process components flow rates.

2. The model which accounts for the thermocouple bead heat balance has been developed.

3. As a result of model validation a method of adjustment thermocouple readings when measuring the temperatures in the presence of strong radial flows has been presented.

4. A set of 3-d models of the experimental natural gas combustion in the down flow reactor VGP-100 B was developed. The models may be used for simulation of the process with a wide range of regime parameters and flow rates variation. 


\section{References}

1. Shannon K.S., Butler B.W. (2003), A review of error associated with thermocouple temperature measurement in fire environments, 2-nd International Wildland Fire Ecology and Fire Management Congress, November 16-20, 2003, Orlando.

2. Pitts W.M., Braun E. (2002), Temperature uncertainties for bear-bead and aspirated thermocouple measurements in fire environments. Thermal measurements: the foundation of fire standards, Dallas.

3. Walker D., Stocks D. (1968), Thermocouple errors in forest fire research, Fire technology, pp. 58-64.

4. Martin R., Cushwa C. (1969), Fire as a physical factor in wildland management, Proceedings of tall timbers fire ecology conference.

5. Sung Chan Kim, Anthony Hamins (2008), On the Temperature Measurement Bias and Time Response of an Aspirated Thermocouple in Fire Environment, Journal of Fire Sciences, 26, pp. 509-529.

6. Peter Struk, Daniel Dietrich, Russell Valentine, Ioan Feier (2003), Comparisons of Gas-Phase Temperature Measurements in a Flame Using Thin-Filament, Pyrometry and Thermocouples NASA/TM, 2003-212096.

7. Z'Graggen A., Friess H., Steinfeld A. (2007), Gas temperature measurement in thermal radiating environments using a suction thermocouple apparatus, Meas. Sci. Technol, 18, pp. 3329-3334.

8. Linda G. Blevins, William M. (1999), Pitts Modeling of bare and aspirated thermocouples in compartment fires, Fire safety Journal, 33, pp. 239-359.

9. Grigorev V.A., Zorin V.M. (1998), Teoreticheskie osnovy teplotekhniki, Moscow.

10. Smith E.J., Natan G.I., Omar N.H., Dally B.B. (2005), Comparison of thermocouple temperature measurements of simple and processing jet propane flames, 5-th Asia-Pacific Conference on Combustion, The University of Adelaide, Australia, 17-20 July 2005, pp. 89-92.

11. Roberts I.L., Coney J.E.R., Gibbs B.M. (2011), Estimation of Radiation Losses from Sheathed Thermocouples, Applied Thermal Engineering, 2011, p. 14

12. Available at: http://web.iitd.ac.in/ prabal/gas-radiation.pdf

13. Isachenko V.P., Osipova V.A., Sukomel A.S. (1975), Teploperedacha, Moscow. 